\title{
Training memory without aversion: Appetitive hole-board spatial learning increases adult hippocampal neurogenesis
}

\author{
Patricia Sampedro-Piquero, Román D. Moreno-Fernández, M. Carmen Mañas-Padilla, Sara \\ Gil-Rodríguez, Ana Luisa Gavito, Francisco J. Pavón, Carmen Pedraza, María García- \\ Fernández, David Ladrón de Guevara-Miranda, Luis J. Santín, Estela Castilla-Ortega
}

Learning experiences are potent modulators of adult hippocampal neurogenesis (AHN). However, the vast majority of findings on the learning-induced regulation of AHN derive from aversively-motivated tasks, mainly the water maze paradigm, in which stress is a confounding factor that affects the AHN outcome. Currently, little is known regarding the effect of appetitively-motivated training on AHN. Hence we studied how spatial learning to find food rewards in a hole-board maze modulates AHN (cell proliferation and immature neurons) and AHN related hippocampal neuroplasticity markers (BDNF, IGF-II and CREB phosphorylation) in mice. The 'Trained' mice were tested for both spatial reference and working memory and compared to 'Pseudotrained' mice (exposed to different baited holes in each session, thus avoiding the reference memory component of the task) and 'Control' mice (exposed to the maze without rewards). In contrast to Pseudotrained and Control mice, the number of proliferating hippocampal cells were reduced in Trained mice, but they notably increased their population of immature neurons assessed by immunohistochemistry. This evidence shows that hole-board spatial reference learning diminishes cell proliferation in favour of enhancing young neurons' survival. Interestingly, the enhanced AHN in the Trained mice (specifically in the suprapyramidal blade) positively correlated with their reference memory performance, but not with their working memory. Furthermore, the Trained animals increased the hippocampal protein expression of all the neuroplasticity markers analyzed by western blot. Results show that the appetitively-motivated hole-board task is a useful paradigm to potentiate and/or investigate AHN and hippocampal plasticity minimizing aversive variables such as fear or stress. 